\title{
Separability and the stella octangula
}

\author{
Åsa Ericsson円 \\ Stockholm University, SCFAB \\ Fysikum \\ S-106 91 Stockholm, Sweden
}

\begin{abstract}
A geometrical picture of separability of $2 \times 2$ composite quantum systems, showing the region of separable density matrices in the space of hermitian matrices, is given. It rests on the criterion of separability given by Peres [1], and it is an extension of the "Horodecki diagram" [2] and the "stella octangula" described by Aravind [3].
\end{abstract}

An illustration of which composite quantum states that are separable is desirable. The separable states is a convex set in the space of hermitian matrices, and a nice geometrical picture of this for Bell-diagonal $2 \times 2$ systems is given by the "Horodecki diagram" [2]. Here we will extend this idea to non-Bell-diagonal mixtures.

A composite quantum system of two subsystems, described by a density matrix $\rho$ in the Hilbert-Schmidt space $\mathcal{H} \otimes \mathcal{H}$, is separable if it can be written as a statistical mixture of product states,

$$
\rho=\sum_{k} w_{k}\left(\rho_{a}\right)_{k} \otimes\left(\rho_{b}\right)_{k},
$$

where $w_{k}$ are positive with $\sum_{k} w_{k}=1$, and where $\rho_{a}$ and $\rho_{b}$ are density matrices for the two subsystems. A necessary criterion for separability, given by Peres [1], is that the partial transpose $\rho^{T_{b}}$ of the density matrix is again a density matrix, i.e. has only non-negative eigenvalues:

$$
\rho_{m \mu, n \nu}^{T_{b}}=\rho_{m \nu, n \mu}, \quad \rho^{T_{b}} \geq 0
$$

This criterion has been shown also to be sufficient for separability of $2 \times 2$ and $2 \times 3$ systems [4].

In the Horodecki diagram the Bell basis states, $\Psi^{ \pm}=|\uparrow \downarrow\rangle \pm|\downarrow \uparrow\rangle$ and $\Phi^{ \pm}=|\uparrow \uparrow\rangle \pm|\downarrow \downarrow\rangle$, are at the vertices of a regular tetrahedron, in which all other states of a $2 \times 2$ system lie [1]. (The cartesian coordinates for a density matrix $\rho$ in the Horodecki diagram is given by the eigenvalues of the correlation tensor $T_{n m}=\operatorname{Tr}\left(\rho \sigma_{n} \otimes \sigma_{m}\right)$. $)$ In the tetrahedron there is an octahedron containing every Bell-diagonal mixture that is separable. Aravind has pointed out that in the Horodecki diagram, Peres' transformation-taking the partial transpose - amounts to a reflection [4]. The reflection of the tetrahedron gives its inverse and the two tetrahedra form a "stella octangula". The region they have in common is the octahedron. Thus the Bell-diagonal states in the octahedron are separable because their partial transpose stays in the tetrahedron. The

\footnotetext{
${ }^{1}$ e-mail: x00aer@physto.se
} 
states outside the octahedron will go to unphysical regions outside the tetrahedron and are therefore non-separable. But non-Bell-diagonal mixtures and their partial transposes depend on additional parameters and can not be satisfactory illustrated in the Horodecki diagram. To get a picture of some of these states we will generalize to similar tetrahedra formed by other basis states than the Bell basis. In these tetrahedra the region of separable states will be found.

The density matrices of $2 \times 2$ systems form a convex set in the 15 real dimensional flat space of all hermitian $4 \times 4$ matrices with unit trace. With the Hilbert-Schmidt distance $D=\sqrt{\frac{1}{2} \operatorname{Tr}\left(\rho_{1}-\rho_{2}\right)^{2}}$ we get unit distance between pure orthogonal states. This means that the density matrices $\rho_{i}, i=1,2,3,4$, of four basis states $|i\rangle$ will sit at the vertices of a regular tetrahedron which spans a 3 -dimensional subspace. The mixtures $\rho=\sum_{i} w_{i} \rho_{i}, \sum_{i} w_{i}=1$, lie within the tetrahedron. As basis states we choose:

$$
\begin{aligned}
& |1\rangle=\cos \alpha|\uparrow \uparrow\rangle+\sin \alpha|\downarrow \downarrow\rangle \\
& |2\rangle=\sin \alpha|\uparrow \uparrow\rangle-\cos \alpha|\downarrow \downarrow\rangle \\
& |3\rangle=\cos \alpha|\uparrow \downarrow\rangle+\sin \alpha|\downarrow \uparrow\rangle \\
& |4\rangle=\sin \alpha|\uparrow \downarrow\rangle-\cos \alpha|\downarrow \uparrow\rangle
\end{aligned}
$$

with $0 \leq \alpha \leq \pi / 4$. These basis states are, for each $\alpha$, equally entangled (i.e. they have the same von Neumann entropy $-\operatorname{Tr}\left(\rho_{a} \ln \rho_{a}\right)$, for the partial traced matrices $\left.\rho_{a}=\operatorname{Tr}_{b} \rho\right)$. For $\alpha=0$ we have the separable product basis states. The entanglement increases with $\alpha$ and for $\alpha=\pi / 4$ we have the maximally entangled Bell basis. $\alpha=\pi / 4$ corresponds to the Horodecki diagram. The generalization here is that $\alpha$, and thus the entanglement, is arbitrary.

When we take the partial transpose we will get a new tetrahedron, consisting of the states $\rho^{T_{b}}=\sum_{i} w_{i} \rho_{i}{ }^{T_{b}}$. The partial transposition is actually given by a reflection in an 11-dimensional plane in the 15-dimensional space. The new tetrahedron will not, except for $\alpha=0$ and $\alpha=\pi / 4$, lie in the same 3 -dimensional subspace as the tetrahedron with the basis states at the vertices. But we will see that there is a plane of fixed points; the two tetrahedra intersect in a 2dimensional plane. The non-separable states will, through the reflection, pass to unphysical regions, i.e. have negative eigenvalues, while the reflection of separable states will give separable states, with non-negative eigenvalues. At the boundary between these regions the determinant of the partially transposed matrix will vanish:

$$
\operatorname{det} \rho^{T_{b}}=0 .
$$

We will use this to find the boundary between separable and non-separable states in the original tetrahedron. Through a straightforward calculation equation (4) is seen to give two possibilities:

$$
\begin{aligned}
& \cos ^{2} \alpha \sin ^{2} \alpha\left(\left(w_{1}-w_{2}\right)^{2}-\left(w_{3}-w_{4}\right)^{2}\right)+w_{1} w_{2}=0 \\
& \cos ^{2} \alpha \sin ^{2} \alpha\left(\left(w_{1}-w_{2}\right)^{2}-\left(w_{3}-w_{4}\right)^{2}\right)-w_{3} w_{4}=0 .
\end{aligned}
$$

Now we introduce the cartesian coordinates (see figure 1a)

$$
x=\frac{1}{2}\left(w_{1}-w_{2}\right), \quad y=\frac{1}{2}\left(w_{3}-w_{4}\right), \quad z=\frac{1}{2 \sqrt{2}}\left(w_{3}+w_{4}-w_{1}-w_{2}\right) .
$$




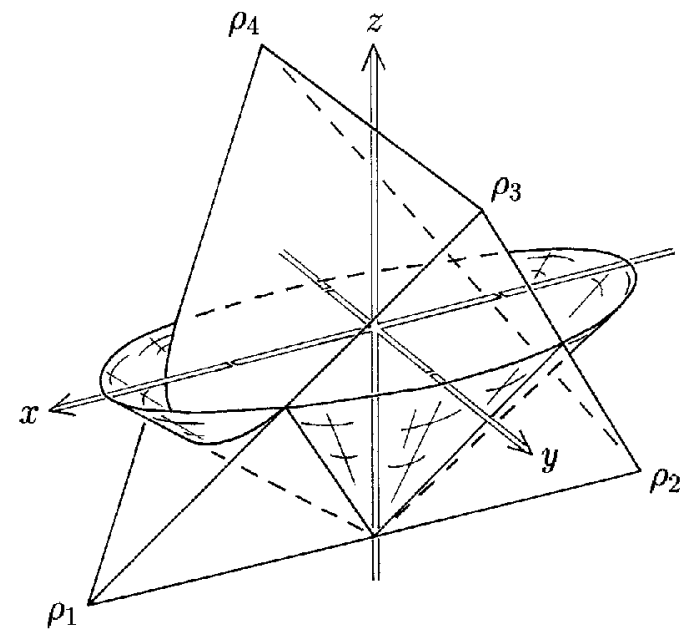

a

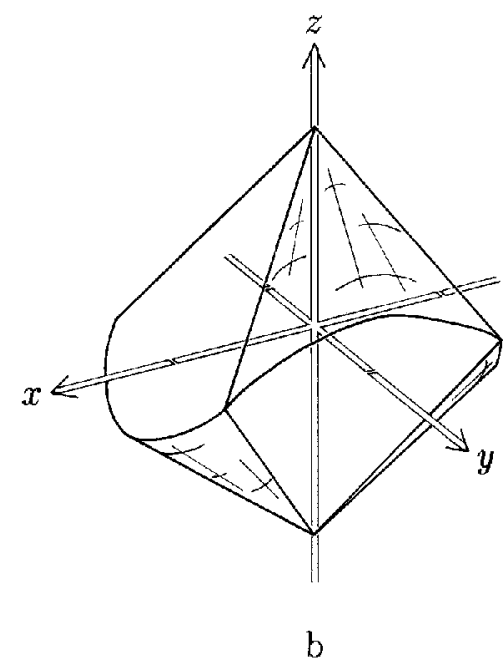

b

Figure 1: a) The tetrahedron with the basis states at the vertices and one of the elliptic cones dividing separable states from non-separable states. b) The region of separable states.

Equations (5) and (6) give the surfaces

$$
\begin{aligned}
& \left(z+\frac{1}{2 \sqrt{2}}\right)^{2}=8 \cos ^{2} \alpha \sin ^{2} \alpha y^{2}+2\left(1-4 \cos ^{2} \alpha \sin ^{2} \alpha\right) x^{2}, \\
& \left(z-\frac{1}{2 \sqrt{2}}\right)^{2}=8 \cos ^{2} \alpha \sin ^{2} \alpha x^{2}+2\left(1-4 \cos ^{2} \alpha \sin ^{2} \alpha\right) y^{2} .
\end{aligned}
$$

For $0<\alpha<\pi / 4$ we have intermediate entangled basis states. Then (8) and (9) are the equations of two elliptical cones with apices in $(0,0, \mp 1 / 2 \sqrt{2})$ and their axes along the z-axis. The cones cut the $x y$-planes in ellipses. For one of the cones the major axis is along the $\mathrm{x}$-axis and for the other along the $\mathrm{y}$-axis (which one depends on $\alpha$ ). In the plane $z=0$ the cones always intersect at the edges of the tetrahedron. These cones together with the sides of the tetrahedron confine the region of all separable states, as shown in figure 1. This region decreases when $\alpha$ increases, i.e. when the entanglement of the basis states increases. For $\alpha=\pi / 8$ we have the special case with circular cones.

When $\alpha=0$ or $\alpha=\pi / 4$ the two cones will degenerate to four planes; the major axes of the ellipses goes to infinity while the minor axes stays finite. For $\alpha=0$ these planes coinside with the four sides of the tetrahedron. Thus every mixture is separable. This is also obvious because the vertices are the product states, which gives $\rho=\sum_{i} w_{i} \rho_{i}$ on the form (1). We have $\rho^{T_{b}}=\rho$; the tetrahedron lies in the plane of reflection.

When $\alpha=\pi / 4$ we have the Bell basis at the vertices and this tetrahedron corresponds to the Horodecki diagram (but the distances differ with a factor $2 \sqrt{2}[2,3])$. The four planes given by equations (8) and (9) are the sides of the inverted tetrahedron and we have obtained the "stella octangula", with the separable states in the octahedron. 
Among the separable states some are fixed points with respect to Peres' transformation, i.e. they fullfill

$$
\rho^{T_{b}}=\rho .
$$

As already noted every point is a fixed point when $\alpha=0$. But for $0<\alpha \leq \pi / 4$ (10) gives $w_{1}+w_{4}=w_{2}+w_{3}=1 / 2$, or in our cartesian coordinates: $x=y$. This plane are the fixed points. In the tetrahedron this is a square. For the Bell basis case taking the partial transpose is a reflection in this plane.

We have met with two properties of the boundary of density matrices mentioned by Harriman [5]: At every boundary point there are some directions in which the boundary is curved, but in other directions it is straight (except for pure states). This is clearly seen in the cases with elliptical cones.

The possibility to illustrate a 15 -dimensional space with 3 -dimensional intersections is of course limited, but these pictures give at least some understanding of how things work. When the entangelment of the basis states at the vertices of the tetrahedron varies we sweep over different intersections and we see how the region of separable states decreases when the entanglement of the basis states increases. For another attempt to illustrate how the separable and non-separable states are located in the set of all density matrices, see [6].

I am grateful to Ingemar Bengtsson for suggesting the problem studied in this paper, for many helpful discussions and for his patience with me.

\section{References}

[1] A. Peres, Phys. Rev. Lett. 77 (1996) 1413.

[2] R. Horodecki and M. Horodecki, Phys. Rev. A 54 (1996) 1838.

[3] P. K. Aravind, Phys. Lett. A 233 (1997) 7.

[4] M. Horodecki, P. Horodecki and R. Horodecki, Phys. Lett A 233 (1996) 1.

[5] J.E. Harriman, Phys. Rev. A 17 (1978) 1249.

[6] F. Verstraete, J. Dehaene and B. De Moor, e-print archive: quant-ph/0107155. 